\title{
The formation and the structure of the intestinal microflora
}

\begin{tabular}{c} 
Sorin Buzinschi \\
Faculty of Medicine, Transilvania University, Brasov, Romania \\
\hline
\end{tabular}

\begin{abstract}
The bacterial population of the adult digestive tract consists mainly of two phyla: Bacteroidetes and Firmicutes. The formation of the intestinal microbiota covers a progressive evolution. In recent years, there is a widely held view that the bacterial colonization begins in utero. The presence of bacteria in the tissue of the placenta, in the foetal membranes, in the amniotic fluid and in the blood of the umbilical cord in healthy foetuses and newborns indicates the early exposure to the bacterial world as a first step of immune training. The colonization of the digestive tract continues and is amplified after birth. The vaginal birth induces an intestinal colonization that reflects the structure of maternal vaginal flora. The intestinal microbiota of $\mathrm{C}$-section newborns is dominated by the maternal skin and oral flora but it also comprises the ecosystem of the skin of the medical personnel and the germs on the hospital surfaces. The breast milk contains live bacteria originating from the mammary canals, areola but especially from the maternal intestine, the latter being a natural source of colonization of the neonatal intestine. The physiological bacterial colonization of the intestine modulates gene expression of the epithelium for immune tolerance; it provides the host with nutrients and contributes to the maturing of the immune system.
\end{abstract}

Keywords: intestine, bacteria, colonization, immunity

The human body and its microbial inheritance are elements of an immense ecosystem in which the bacterial population contains 100 times more genes and 10 times more cells than the human ones $(1,2)$. Bacteria are unicellular organisms, prokaryotes, whose genetic material is not protected by a nuclear membrane. They differ from eukaryotes in the following components: the cell wall and plasma membrane, the colloidal cytoplasm, the ribosomes (protein synthesis), the genetic information of a circular chromosome (nucleosome) and the plasmids (they contain double-stranded DNA), multiplication usually by direct division. Taxonomically speaking, they differ in the great domains, phyla, classes, orders, families, genera, species and subspecies (2). In practice, this taxonomic hierarchy is inoperative. In scientific papers, we will rather encounter references to bacterial species or subspecies, while the enumeration of a long series of taxonomic classes is avoided. For the digestive system, at least, there are two important phyla: Bacteroidetes and Firmicutes. These form the dominant flora in western populations; other phyla represent
$10 \%$ of the microbiome (Proteobacteria (4.5\%), in small amounts Escherichia, Helicobacter and Actinobacter genera (3). The normal microbial colonization of the human body depends on the local chemical environment conditions, on the oxygenation level, on the nutrient intake of the host tissue and on the intervention of the immune system. Some species proliferate by using the local nutrient circumstances, adhering to the niches of different organs and, due to their continuity, they constitute the basis of a stable population. As a whole, the microorganisms that colonize the surfaces and some of the cavities of the body form the local microbiota. The formation of the intestinal microbiota covers a progressive evolution. Thus, we are able to outline a prenatal stage, a phase of exclusive breastfeeding or milk formulas, changes in microflora after the introduction of solid foods and a formation of the stable microbiota.

\section{The bacterial colonization in utero}

According to acknowledged opinions, the amniotic environment is considered to be sterile in nor- 
mal, full-term pregnancies. However, in the animal world, the transmission of the maternal microbiome to the foetus is considered to be a common phenomenon. The vertical transmission of the human maternal microbiome in the placenta and in the foetal meconium suggests that the colonization process begins before birth. Along with the neurological development, the foetus ingests amniotic fluid, especially during the third semester of pregnancy, which represents the beginning of the colonization. The presence of bacteria in the placental tissue, in the foetal membranes, in the amniotic fluid and in the blood of the umbilical cord in healthy foetuses and newborns, without infection or inflammatory signs, suggests the early exposure to the bacterial world as a first step of immune training $(4,5)$. The microbial flora of 320 sterilecollected placental specimens has been investigated by Aagard et al by using 16SrRNA gene sequencing correlated with similar investigations of the oral, skin, respiratory airways (nasal), vaginal microflora and of the intestinal microbiome of pregnant women (4). The author specifies the low biomass of placental microbiome (0.002 DNA mg was extracted from $1 \mathrm{~g}$ of placental tissue). The profile of the niche of the studied placental microbiome encompassed the following genera of nonpathogenic commensal strains of bacteria: Firmicutes, Tenericutes, Proteobacteria, Bacteroidetes and Fusobacteria $(4,5)$. Current paradigms state that most of the intrauterine infections that triggered premature birth originate in the inferior genital tract due to the ascent of the germs in the sterile intrauterine environment. However, numerous bacterial classes that were locally detected have not stemmed from the urogenital tract, but they were commensal species from the oral cavity of the pregnant woman (4). Some of these germs such as Fusobacterium nucleatum (oral anaerobe, gramnegative) spread hematogenously, functions as a pathfinder for other ordinary commensals such as $E$. coli strains. Surprisingly, among the bacterial species found in the placenta, E. coli has been one of the most abundant strains. While reviewing the data that indicate the mother-to-foetus transmission of bacterial communities, Keeler and Weilkamp (6) have highlighted the bacterial commensal colonization of the meconium 2 hours after birth even before feeding and the presence of microbial population in segments of excised intestine right after birth in the case of some malformations. Commensal bacteria of the swallowed amniotic fluid contribute to the maturing of the fetal intestine, to the development of the immune system and to the pro- tection by competition against the potential pathogens.

The bacterial uterine colonization under physiological conditions of non-ongoing pregnancy can be divided into resident germs, "tourists" and invaders. Some data invalidate the existence of a stable uterine population which would play the role of maintaining the local hemostasis. In contrast with the paradigm of the sterile womb environment, the molecular identification techniques of the bacterial communities have indicated the acquisition, during pregnancy, of a microbiome, which, as shown in the study, contributes to the creation of immune responses, to the structural and metabolic relations with the host. Some believe that the intrauterine colonization hypothesis is unfounded due to the limitations of molecular methods on the study of minimal biomass, due to the lack of an adequate contamination control and to the incapacity of proving the bacterial validity.

The abundant presence of bacteria in the amniotic fluid determines chorioamnionitis, the local growth of leucocytes, of proinflammatory cytokines, which represents a cause of the premature birth (7). Bacteria which produce vaginal, urinary infections or infections of the amniotic fluid trigger the production of antimicrobial peptides and of the cytokines that are involved in premature birth and/ or early membrane rupture. Although infections represent important triggering factors, the alteration of the inflammatory cascade by polymorphisms of the genes TLR4, IL-1 $\beta$, IL-4, IL-10, IL1 ra and TNF- $\alpha$ can be considered one of the genetic premises of the premature birth (8). The rise of the prematurity degree is correlated with a high level of proinflammatory cytokines in the amniotic fluid, with the abundant development of the microbiota of the meconium and with the structure of the microbial community (9). The Enterobacter, Enterococcus and Lactobacillus strains are negatively linked with the gestational age due to the inflammatory response that they trigger (9).

The traditional culture germ-identification methods provide incomplete data on the bacterial populations of the human microbiome. Metagenomic techniques and sequencing technology have revolutionized our understanding of the microbial populations. The sequencing of a ribosomal gene (16SrRNA), distinctively conserved in prokaryotes (bacteria), allows the differentiation between species and the identification of some that could not be cultivated. Although current paradigms state that intrauterine infections which trigger preterm birth are due to the germs of the inferior genital tract, 
owing to new technology, the most frequently identified agents are the oral cavity commensals (Fusobacterium nucleatum, gram-negative, anaerobe and others), hematogenously spread. Han et al, J Clin Microbiol, 2009,47,1,38-47

The immune system plays the role of controlling the microbiota development during the perinatal period in order to achieve full term pregnancy and to control the antigens for the state of imunotolerance creation.

\section{The neonatal intestinal microbiome}

The colonization of the digestive tract initiated during intrauterine life is continued and rapidly amplified after birth. Numerous factors determine the composition of the microbiota in the first stages of life: type of birth (vaginal/C-section), natural/ artificial nutrition, environment, hygiene and the use of antibiotics. The vaginal microbiota passes through important changes during pregnancy. Although the composition of the isolated bacterial strains differs in some studies, the vaginal microbiome seems to be dominated by species such as $L a c-$ tobacillus, Clostridium and Actionomicetes (4)(5) (10). Among these, due to the production of lactic acid, Lactobacillus strains lower the local $\mathrm{pH}$ (below 4.5), having the effect of protecting against the colonization with other species, especially pathogenic ones (10). Compared to the first semester of pregnancy, the decrease of intestinal microbial diversity of the pregnant woman in the $3^{\text {rd }}$ semester suggests changes similar to those of the metabolic syndrome in which obesity and insulin resistance have been correlated with the structure of intestinal microbiota (11). In contrast with the negative effects of obesity, the increase in adiposity and the decrease in insulin sensitivity are beneficial during the pregnancy due to the fact that they provide the foetus with more glucose and prepare the body for the energy requirements of lactation (11). Vaginal birth induces an intestinal colonization which reflects the structure of the maternal vaginal flora, dominated by Lactobacillus and Prevotella species, the first representing $50 \%$ of the entire bacterial ecosystem (12). Together with the vaginal flora, the maternal fecal flora contributes to the diversity of colonization with Lactobacillus, Bifidobacterium, E.coli and Enterococcus. During the first week of life, the microbiome of the full-term newborn is rapidly colonised with species of the phyla of Actinobacteria (including Bifidobacterium), Proteobacteria, Bacteroidetes and fewer of Firmicutes $(4,13)$. In the case of preterm birth, under 1,200 g, the intestinal microbiome is dominated by Firmicutes and Tenericutes genera and less by Actinobacteria $(4,5)$.

The intestinal ecology in preterm newborns is characterised by a lower germ density and diversity due to the type of birth, hospitalization and nutrition. This is a matter of great importance as the primary intestinal colonization represents the first immune training of the child. The colonization with maternal germs allows the communication with these, the expression of some genes in the intestinal epithelium and the initiation of the immune circuits. Unlike vaginal births, the microbiota of Csection newborns is dominated by the maternal oral and skin flora, by Propionilbacterium, Corynebacterium and Streptococcus (10), showing a low colonization of anaerobes such as Bacteroides and Bifidobacterium (13). Their microbiome also reflects the skin ecosystem of the medical personnel, the microbial surface colonization etc., which generates the risk of intestinal colonization with antibiotic-resistant germs. A high proportion of bacterial antibiotic-resistant genes has been identified within their intestinal population. The methicillin-resistant strains of Staphylococcus aureus [MRSA] (present in $64-82 \%$ of these) would explain the skin infections with this germ (10). The antibiotic treatment before or during the birth modulates the neonatal intestinal ecosystem by altering the ratio between gram-positive and gram-negative germs, and, possibly, by inducing bacterial antibiotic resistance. The intestinal colonization polymorphism may explain the great differences in certain published studies concerning the structure, ratio and the evolution of the neonatal microbiota. The first colonizing bacteria structured gene expression in the intestinal epithelial cells, creating themselves a favourable habitat to the detriment of the following inhabitants (13). Mention should be made that the birth flora influences the diversity and the functioning of the intestinal microbiome months, years after birth or even the flora in adults.

\section{Natural nutrition and the intestinal colonization}

The human milk ensures the healthy and optimal development of the child. Apart from the food principles that provide nutrition for the newborn, the maternal milk also contains many types of oligosaccharides, neutrophils, macrophages, $\mathrm{T}$ cells and $\mathrm{B}$ cells but also live bacteria. Some of these originate from mammary ducts or areola, while some are intrinsic components of the milk and originate from the maternal intestinal microbiome 


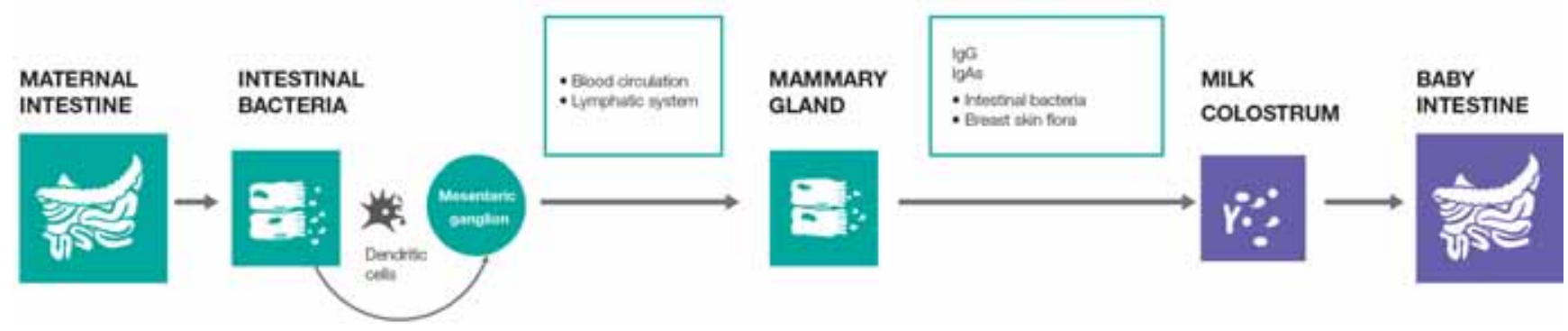

FIGURE 1. The immune information transfer from the maternal intestine to the intestine of naturally breastfed newborn

(14-16). The assertions that the maternal milk is not sterile, even when it is aseptically collected, support the idea that it is a source of natural bacterial colonization of the neonatal intestine due to the fact that identical DNA fragments may be identified in the mother's fecal matter, blood, milk and in the infant's stools (15). In the case of natural nutrition, the bacterial transmission through breast milk represents a colonization method of the neonatal intestine with a flora similar to the maternal one, allowing the circulation of the germs through the mammary-enteral pathway.

The transfer of viable bacteria or their genetic material is ensured by mononuclear maternal cells both during the pregnancy and after birth $(6,14)$. The maternal weight influences the intestinal microbiota and the composition of flora transmitted through milk (10). In obese pregnant women, the abundance of $S$. aureus strains has been noticed during the first 6 months of lactation, while during the first month, the abundance of Lactobacillus strains was observed (10). The maternal microbiome is influenced by genetic and immunological factors, diet and medication, elements which determine the structure of the intestinal flora and the configuration of the germs transferred to the infant (6). The intestinal colonization of the newborn and of the infant is more dependent on the breast tegument (for Staphylococcus, Corynebacteria and other cutaneous bacteria such as Propionilbacteria), on the maternal oral cavity (Fusobacterium nucleatum, Bregeyella spp, Porphyromonas gingivalis etc.) (17). In a study of 1032 infants aged 1 month, Penders et al (18) have found that the C-section newborns had an intestinal flora poor in Bifidobacteria and Bacteroides, being more frequently colonised by $\mathrm{Cl}$ difficile, unlike those who went through vaginal birth. The infants who were fed only with milk formulas are more frequently colonized with E.coli, Cl difficile, Bacteroides and Lactobacillus. Hospitalization and prematurity increase the colonization of $\mathrm{Cl}$ difficile strains, while the antibiotic treatment decreases the population of Bifidobacteria and Bacteroides (18). The differences between breastfed infants and those nourished with milk formulas do not narrow down to the composition of intestinal flora; they also refer to the protective effects they have on the newborns.

TABLE 1. The differences between the intestinal colonization of the naturally fed infants and those artificially fed and the immune protection they provide $(13,18,19)$

\begin{tabular}{|l|l|}
\hline Naturally fed infants & Artificially fed infants \\
\hline $\begin{array}{l}\text { The diges ve tract is } \\
\text { primarily colonised } \\
\text { by Bifidobacteria, } \\
\text { Bacteroides }\end{array}$ & $\begin{array}{l}\text { The diges ve tract is colonised } \\
\text { by a diverse flora which } \\
\text { comprises E.coli, Cl difficile, } \\
\text { Bacteroides, Prevotella and } \\
\text { Lactobacillus }\end{array}$ \\
\hline $\begin{array}{l}\text { Human milk contains } \\
\text { numerous an microbial and } \\
\text { immunomodula ng factors } \\
\text { which limit the growth of } \\
\text { the pathogens, live cells } \\
\text { (neutrophils, macrophages, } \\
\text { Tand B lymphocytes) }\end{array}$ & $\begin{array}{l}\text { Complex microbial flora; high } \\
\text { propor on of anaerobes } \\
\text { (faculta ve/obligate) such } \\
\text { as Enterobacteria, Streptococcus } \\
\text { and Cl difficile }\end{array}$ \\
\hline
\end{tabular}

The intestinal colonization with maternal microbiota is optimal in vaginal home birth and under natural nutrition circumstances. In maternity wards of the hospitals, diverse colonisation with longterm effects on the infant and newborn microbiome may occur. Along with the diversification and the introduction of solid food, changes in the intestinal microbiota take place due to the development of some species dominant in adults (Bacteroidetes, Firmicutes and Clostridium) (10). The alterations of the flora depend on the evolution of the nutritional substrate, which is a source of commensal species that extract energy, produce vitamins, shortchain fatty acids and amino acids.

Once formed, the intestinal microbiome establishes symbiotic relationships with its host due to the processing of the undigested substrates, the nutrient limitation for the pathogens and due to the space competition with the latter, the formation of the immune tolerant state between bacteria and the immune system, the training and the development 
TABLE 2. The intestinal microbiota in infants and the commensal or symbiotic relation with its host. Review of the species considered to be important $(10,13,19,20,21)$.

\begin{tabular}{|c|c|}
\hline $\begin{array}{l}\text { Species of the intestinal } \\
\text { flora }\end{array}$ & Colonization \\
\hline Bifidobacteria & $\begin{array}{l}\text { They abundantly colonize the diges ve tract of newborns especially with } B \text { bifidus, } B \text { breve, } B \text { infan } s \text {. } \\
\text { They are gram-posi ve germs, anaerobic bacteria; their localiza on is closely connected with the mucous } \\
\text { membrane of the epithelium }\end{array}$ \\
\hline Lactobacillus & $\begin{array}{l}\text { Gram-posi ve germs colonize especially the colon. In the newborn intes ne, they come from maternal } \\
\text { vaginal flora and from the maternal milk. L acidophilus, is the most common species of Lactobacillus a er } \\
\text { bifidobacteria, in terms of importance regarding the intes ne of naturally fed infants. Adhesion to the } \\
\text { intes nal epithelium provides them with protec ve proper es against the coloniza on with pathogens. }\end{array}$ \\
\hline Clostridium & $\begin{array}{l}\text { They are gram-posi ve germs, obligate anaerobes, spore-forming. They physiologically colonise the } \\
\text { intes nal tract but have the ability to elaborate exotoxins ( } \mathrm{A} \text { and } \mathrm{B}) \text {, due to this being pathogens and poten al } \\
\text { pathogens due to spore forma on. Among the Clostridium species, } \mathrm{Cl} \text { difficile colonizes the intes ne of the } \\
\text { infant without producing harmful effects under normal condi ons. Most of the infants colonized with } \mathrm{Cl} \\
\text { difficile are asymptoma } \mathrm{c} \text { and manifest IgG an bodies against } \mathrm{A} \text { and } \mathrm{B} \text { toxins which provide them with long- } \\
\text { term immunity. }\end{array}$ \\
\hline Bacteroides & $\begin{array}{l}\text { They are gram-negative germs, obligate anaerobes, commensal and conditioned pathogens. They represent } \\
\text { approximately } 30 \% \text { of the bacterial mass of the intestine. They possess mutual relations with the host by the } \\
\text { decomposition of vegetal substrates. They develop abundantly in the digestive tract; the high protein and } \\
\text { animal fat intake encourages its colonization, while the consumption of mainly carbohydrates encourages } \\
\text { the development of Prevotella species. Bacteroides fragilis, anaerobe, generally commensal, is part of the } \\
\text { colonic flora. It makes up } 0.5 \% \text { of the bacteria excreted present in the stool. It is involved in infections with } \\
\text { anaerobes originating from the intestinal flora. }\end{array}$ \\
\hline Escherichia coli & $\begin{array}{l}\text { It is a gram-negative germ, a facultative anaerobe. It colonizes early the intestine of the infant. Maternal milk } \\
\text { has a protective effect against it due to the inhibition of colonization. Some species are commensal, they take } \\
\text { part in the synthesis of vitamins K and B, they stimulate IgA secretion in lymphocytes. } \\
\text { Strains such as O157:H7, verotoxin secretors, can produce severe effects such as hemorrhagic enteritis and } \\
\text { haemolytic-uremic syndrome. }\end{array}$ \\
\hline
\end{tabular}

of systemic immunity. The disturbances of the formation and the preservation of the intestinal micro- biota may constitute risk factors in the inflammatory and metabolic pathology of the adult.

\section{Conflict of interest: none declared} Financial support: none declared

\section{$\overline{\text { REFERENCES }}$}

1. Todar K., in Todar's Online Textbook of Bacteriology http:// textbookofbacteriology.net/bacteriology_3.html

2. Brenner D., Staley J., Krieg N. Classification of Prokaryotic Organisms and the Concept of Bacterial Speciation in Bergey's Manual of Systematic Bacteriology, Vol. 2, Springer, 2004.

3. ${ }^{* * *}$ Human Microbiome Project. Structure, Function and Diversity of the Healthy Human Microbiome in Nature, 2012, No. 486, pp. 207-214.

4. Aagard K., Ma J., Antony K. et al. The Placenta Harbors a Unique Microbiome in Science Translational Medicine, 2014, Vol. 6, Issue 237.

5. Aagard K., Riehle K., Ma J. et al. A metagenomic approach to characterization of the vaginal microbiome signature în pregnancy in PloS One, 2012,7,6,e36466.

6. Romano-Keeler J., Weitkamp J-H. Maternal influences on fetal microbial colonisation and immune development in Pediat Res, 2015, Vol. 77, 1-2, pp.189-195.

7. Putignani L., Del Chierica F., Petrucca A. et al. The human microbiota: a dynamic interplay with the host from birth to senescence settled during childhood in Pediatric Research, 2014, Vol. 76,1, pp.2-10.

8. Nesin M. Genetic Basis of Preterm Birth in Frontiers Bioscience, 2007, Vol.12, pp.115-124.

9. Ardissone A., de la Cruz D., Davis-Richardson A. et al. Meconium microbiome analysis identifies bacteria correlated with premature birth in PloS One, 2014,10,9,3, e90784.

10. Nuriel-Ohayon M., Neuman H., Koren O. Microbial Changes during Pregnancy, Birth, and Infancy in Frontiers Microbiol, 2016, V.7, 1031.
11. Koren O., Goodrich J., Cullender T. et al. Host remodeling of the gut microbiome and metabolic changes during pregnancy in Cell, 2012, Vol.150,3, pp.470-480.

12. Baker J., Chase D., Herbst-Kralovetz C. Uterine microbiota: Residents, Tourists, or Invaders? in Front Immunol, 2018, 9, 208.

13. Gritz E., Bhandari V. The human neonatal gut microbiome: a brief review in Frontiers Pediat, 2015, 3, Article 17.

14. Palmer C., Bik E., DiGiuglio D. et al. Development of the Human Infant Intestinal Microbiota in PloS Biology, 2007,5,7,e177.

15. Perez P., Dore J., Leclerc M. et al. Bacterial Imprinting of the Neonatal Immune System: Lessons From Maternal Cells? in Pediatrics, 2007,119,e724.

16. Parigi A., Eldh M., Larssen P. et al. Breast milk and solid food shaping intestinal immunity in Frontiers Immunol, 2015, 6, doi: 10.3389/fimmu.2015.00415.

17. Langhendries J. Microbiota in early infancy in Pediatrics, 2006, 118, 2, 511-521.

18. Penders J., Thjis C., Vink C. et al. Factors influencing the composition of the intestinal microbiota in early infancy. Pediatrics, $2006,118,2,511-521$

19. * * Newborn baby digestive tract. https:// microbewiki.kenyon. edu/ index.php/ Newborn_baby_digestive_tract

20. Todar K. The Normal Bacterial Flora in Humans in Todar's Online Texbook of Bacteriology, 2012.

21. Arboleya S., Watkins C., Stanton C. et al. Gut Bifidobacteria Populations în Human Health and Aging in Front Microbiol, 2016, journal.trontiersin.org/ article/ 10.3389/fmicb. 